\title{
Die mesoklimatische Verteilung von Lufttemperatur und Nieder- schlag über der hocharktischen Tundra von Axel Heiberg Island, N.W.T., Kanada
}

\section{Einleitung}

Die Arktis kann in Meeres-, Tundra- und Gletscheroberflächen unterteilt werden, die sich inbezug auf Strahlungs-, Wärme und hydrologische Charakteristiken deutlich voneinander unterscheiden. Diese Oberflächeneigenschaften bestimmen zusammen mit der Topographie und der großräumigen atmosphärischen Zirkulation die Bildung der regionalen Klimate in der Arktis. In den Gebieten nördlich von $70^{\circ} \mathrm{N}$, die 15,6 Millionen $\mathrm{km}^{2}$ oder $3 \%$ der ganzen Erdoberfläche einnehmen, befinden sich 17\% Land ohne ständige Eisdecke, $75 \%$ sind Meere und die restlichen $8 \%$ entfallen auf Gletscher. Die gegenwärtig eisfreie Landoberfläche besteht $\mathrm{zu} 12 \%$ aus borealen Nadelwäldern und zu $88 \%$ aus Tundra.

Seit dem ersten internationalen Polarjahr (1882-1883) sind verschiedene Aspekte des Tundrenklimas erforscht worden. Dennoch ist die gegenwärtige Kenntnis der mittelmaßstäblichen Verteilung der grundlegenden Klimaelemente noch nicht ausreichend bekannt. Die meisten arktischen Wetterstationen liegen an der Küste. Stationen im Landesinnern und besonders in größeren Höhen existieren faktisch nicht. JACK. SON (1959) zeigte, daß das Klima bei Hazen-Camp im Innern von Ellesmere Island so verschiedenartig ist, $\mathrm{da} \beta$ es unmöglich wird, es anhand von Daten von Alert und/oder Eureka einzustufen. Das Bedürfnis nach mehr Fallstudien ist offensichtlich: daher die vorliegende Studie. Eine umfassende Studie der Tundra im Expeditionsgebiet berücksichtigte sowohl die Energie- und Wasserbilanzen als auch das Klima über und an der Oberfläche, in der Schneedecke und im Boden. Die gegenwärtige Untersuchung behandelt aber nur die Luftemperatur im Wetterhüttenniveau und den Niederschlag.

Die vorliegende Studie ist ein Teilprojekt der langjährigen Axel Heiberg Island Expeditionen der MacGill Universität in Montreal (MÜLLER et al., 1961 und 1963) und der ETH Zürich.

\section{Die klimatologischen Stationen und ihre Umgebung}

Das Forschungsgebiet (Fig. 1) umfaßt die rund $40 \mathrm{~km}^{2}$ Tundraoberfläche im generell südexponierten $\mathrm{Ge}$ lände des Expedition River Valley zwischen dem
White Glacier im Osten und dem Expedition Fiord im Westen. Im Norden ist es von Gletschern begrenzt und im Süden vom Expedition River. Die Höhenerstreckung reicht vom Meeresspiegel bis auf $800 \mathrm{~m}$ ü. M., d. h. bis etwa 100 bis $200 \mathrm{~m}$ unter die mittlere Gleichgewichtslinie der umliegenden Gletscher. In diesem Gebiet wurden neun klimatologische Stationen errichtet. Ferner wurden fünf Stationen auf den benachbarten Gletschern unterhalten sowie eine weitere auf einer ganz von Gletschern umgebenen Tundraenklave weiter im Osten. Die Daten dieser zusätzlichen Stationen wurden gebraucht, um Vergleiche zwischen Tundra und Gletscher anzustellen.

Die Station am Fjord sollte das Klima an der Küste wiedergeben und damit die Beziehung zu den offiziellen permanenten Wetterstationen der Regierung herstellen. Die Station auf dem Sander vor dem White Glacier wurde 1962 aufgestellt (MÜLLER und ROSKINSHARLIN, 1967), um das Klima nahe der Gletscherzunge zu erfassen. Die Expedition River Station liegt $90 \mathrm{~m}$ ü. M. und ist zusammen mit dem Base Camp am weitesten vom Fjord und zugleich den Gletschern entfernt. Am Base Camp lag die Hauptstation, die während der ganzen Forschungsperiode betrieben wurde. Es liegen bereits synoptische Beobachtungen über eine Periode von 18 Jahren vor. Die Wolf River Station liegt auf einer Höhe von $600 \mathrm{~m}$ ü. M. in einem Talboden des oberen Einzugsgebietes des Wolf River. Sie ist vor dem raschen Austausch von Luftmassen und ebenso vor Sonnenschein geschützt. Die Ermine Ridge Station befindet sich auf der Kulmination eines Grates in einem typisch arktisch-alpinen Felsenmeer. Die Schneedecke hält sich auf dieser Höhe bis Juli und in einzelnẹn Jahren bis weit in den August hinein. Die Pflanzendecke ist hier, verglichen mit den anderen Stationen, am dürftigsten.

\section{Die Lufttemperatur im Wetterhüttenniveau}

Die Meßwerte der Luftemperatur von drei Jahren sind in Tab. 1 zusammengefaßt. Berücksichtigt wurden Monatsmittel, Monatsmittelwerte der Tagesmaxima und -minima, Tagesschwankung, tägliche Amplitude und aperiodische Tagesvariation. Diese Größen

Atsumu Ohmura und Prof. Dr. Fritz Müller, Geographisches Institut, ETHZ, Sonneggstraße 5, 8006 Zürich. 
Fig. 1: Standorte der Instrumente, Axel Heiberg Island.

Die Stationnummer entspricht derjenigen in Tab. 1.

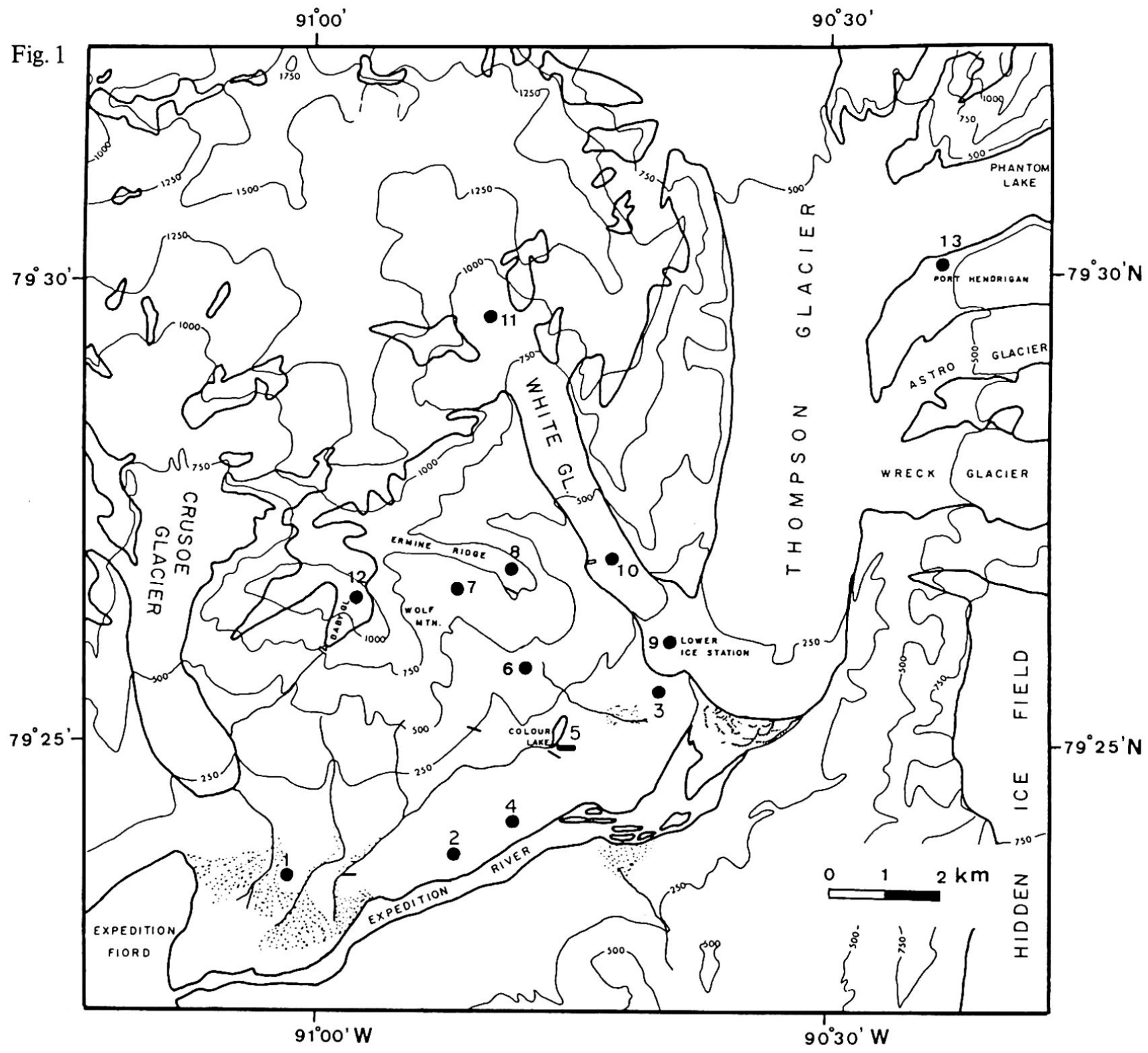

stellen ein Minimum dar, um die verschiedenen Klimalokalitäten charakterisieren zu können. In Fig. 2 wird die Höhenabhängigkeit des Monatsmittels der Lufttemperatur und des zugehörigen Monatsmittels der täglichen Amplitude gezeigt. Zum Vergleich wurde das Monatsmittel der Luftemperatur in den Standarddruckniveaus über Isachsen beigefügt.

Das wichtigste Merkmal der Mitteltemperaturverteilung im Mai ist deren Homogenität, die durch das Fehlen eines vertikalen Temperaturgradienten in der freien Atmosphäre erklärt werden kann. Die relativ homogenen Eigenschaften der Schneedecke, die das ganze Gebiet - mit Ausnahme der windexponierten Felspartien - bedeckt, trägt ebenfalls zu dieser bemerkenswerten horizontalen und vertikalen Gleichförmigkeit der Temperatur bei. Eine weitere Eigenheit der Luftemperatur ist, daß sie auf allen Niveaus beständig höher liegt als die Temperatur der freien Atmosphäre. Für den gesamten untersuchten Höhenbereich beträgt die durchschnittliche Differenz $3,2^{\circ} \mathrm{C}$. 
Tabelle 1: Monatsmittel von Tagesmittel, Tagesmaximum, Tagesminimum, Tagesbereich, täglicher Amplitude und aperiodischer Tagesvariation für Stationen im Expedition Gebiet, Axel Heiberg Island, N.W.T., Canada, 1969, 1970 und 1972.

\begin{tabular}{|c|c|c|c|c|c|c|c|c|c|c|c|c|}
\hline & Stationen & Fiord & $\begin{array}{r}\text { Outwash } \\
\text { Plain }\end{array}$ & $\begin{array}{r}\text { Expedition } \\
\text { River }\end{array}$ & $\begin{array}{r}\text { Base } \\
\text { Camp }\end{array}$ & $\begin{array}{r}\text { Port } \\
\text { Hendrigan }\end{array}$ & $\begin{array}{l}\text { Wolf } \\
\text { River }\end{array}$ & $\begin{array}{r}\text { Ermine } \\
\text { Ridge }\end{array}$ & $\begin{array}{r}\text { Lower } \\
\text { Ice }\end{array}$ & $\begin{array}{r}\text { Anniversary } \\
\text { Profile }\end{array}$ & $\begin{array}{c}\text { Morain } \\
\text { Profile }\end{array}$ & $\begin{array}{r}\text { Baby } \\
\text { Glacier }\end{array}$ \\
\hline & Station Nr. & 1 & 3 & 4 & 5 & 13 & 7 & 8 & 9 & 10 & 11 & 12 \\
\hline & Höhe in m & 10 & 50 & 90 & 200 & 400 & 600 & 800 & 200 & 330 & 870 & 1050 \\
\hline Monatsmittel & $\begin{array}{l}\text { Mai } \\
\text { Juni } \\
\text { Juli } \\
\text { August }\end{array}$ & $\begin{array}{r}-9,6 \\
-\quad 0,4 \\
4,8 \\
3,9\end{array}$ & $\begin{array}{r}-10,9 \\
-\quad 0,3 \\
4,5 \\
3,9\end{array}$ & $\begin{array}{r}-10,8 \\
-\quad 0,2 \\
5,3 \\
4,6\end{array}$ & $\begin{array}{r}-10,6 \\
-\quad 0,8 \\
4,4 \\
3,8\end{array}$ & $\begin{array}{r}-2,0 \\
2,4 \\
2,4\end{array}$ & $\begin{array}{r}-10,0 \\
-\quad 1,6 \\
2,2 \\
2,9\end{array}$ & $\begin{array}{r}-9,1 \\
-\quad 1,1 \\
1,6 \\
2,4\end{array}$ & $\begin{array}{r}-10,5 \\
-\quad 1,5 \\
2,9 \\
2,9\end{array}$ & $\begin{array}{r}-10,5 \\
-\quad 2,5 \\
1,5 \\
1,8\end{array}$ & $\begin{array}{l}-10,2 \\
-\quad 3,9 \\
-0,2 \\
-0,7\end{array}$ & $\begin{array}{l}-8,2 \\
-\quad 2,8 \\
-\quad 0,8 \\
-\quad 1,3\end{array}$ \\
\hline $\begin{array}{l}\text { Monatsmittel } \\
\text { Tages- } \\
\text { maximum }\end{array}$ & $\begin{array}{l}\text { Mai } \\
\text { Juni } \\
\text { Juli } \\
\text { August }\end{array}$ & $\begin{array}{r}-5,0 \\
2,1 \\
7,4 \\
6,4\end{array}$ & $\begin{array}{r}-7,4 \\
1,4 \\
6,7 \\
6,1\end{array}$ & $\begin{array}{r}-6,5 \\
1,8 \\
7,2 \\
6,5\end{array}$ & $\begin{array}{r}-6,6 \\
1,7 \\
7,1 \\
6,5\end{array}$ & $\begin{array}{l}0,0 \\
4,0 \\
4,9\end{array}$ & $\begin{array}{r}-7,1 \\
0,4 \\
4,3 \\
5,0\end{array}$ & $\begin{array}{r}-4,5 \\
2,7 \\
4,3 \\
4,7\end{array}$ & $\begin{array}{r}-7,4 \\
0,7 \\
4,8 \\
4,8\end{array}$ & $\begin{array}{r}-7,6 \\
-0,4 \\
3,3 \\
3,6\end{array}$ & $\begin{array}{r}-6,8 \\
1,2 \\
3,2 \\
3,1\end{array}$ & $\begin{array}{l}0,7 \\
1,7 \\
0,8\end{array}$ \\
\hline $\begin{array}{l}\text { Monatsmittel } \\
\text { Tages- } \\
\text { minimum }\end{array}$ & $\begin{array}{l}\text { Mai } \\
\text { Juni } \\
\text { Juli } \\
\text { August }\end{array}$ & $\begin{array}{r}-14,0 \\
-\quad 2,6 \\
2,3 \\
1,4\end{array}$ & $\begin{array}{r}-14,5 \\
-\quad 2,1 \\
2,2 \\
1,2\end{array}$ & $\begin{array}{r}-15,2 \\
-\quad 2,9 \\
2,2 \\
1,0\end{array}$ & $\begin{array}{r}-14,6 \\
-\quad 3,3 \\
1,3 \\
0,7\end{array}$ & $\begin{array}{r}-4,0 \\
0,8 \\
-\quad 0,1\end{array}$ & $\begin{array}{r}-12,8 \\
-\quad 3,7 \\
0,3 \\
1,0\end{array}$ & $\begin{array}{r}-13,2 \\
-\quad 3,9 \\
-\quad 0,7 \\
0,4\end{array}$ & $\begin{array}{r}-13,6 \\
-\quad 3,5 \\
0,9 \\
0,3\end{array}$ & $\begin{array}{r}-14,0 \\
-\quad 4,5 \\
0,1 \\
0,5\end{array}$ & $\begin{array}{l}-16,1 \\
-\quad 8,0 \\
-\quad 3,6 \\
-4,2\end{array}$ & $\begin{array}{l}-5,5 \\
-2,6 \\
-\quad 2,9\end{array}$ \\
\hline $\begin{array}{l}\text { Monatsmittel } \\
\text { Tagesbereich }\end{array}$ & $\begin{array}{l}\text { Mai } \\
\text { Juni } \\
\text { Juli } \\
\text { August }\end{array}$ & $\begin{array}{l}9,0 \\
4,7 \\
5,1 \\
5,0\end{array}$ & $\begin{array}{l}7,1 \\
3,5 \\
4,5 \\
4,9\end{array}$ & $\begin{array}{l}8,7 \\
4,7 \\
5,0 \\
5,5\end{array}$ & $\begin{array}{l}8,0 \\
5,0 \\
5,8 \\
5,8\end{array}$ & $\begin{array}{l}4,0 \\
3,2 \\
5,0\end{array}$ & $\begin{array}{l}5,7 \\
4,1 \\
4,0 \\
4,0\end{array}$ & $\begin{array}{l}8,7 \\
6,6 \\
5,0 \\
4,3\end{array}$ & $\begin{array}{l}6,2 \\
4,2 \\
3,9 \\
4,5\end{array}$ & $\begin{array}{l}6,4 \\
4,1 \\
3,2 \\
3,1\end{array}$ & $\begin{array}{l}9,3 \\
9,2 \\
6,8 \\
6,3\end{array}$ & $\begin{array}{l}5,5 \\
6,2 \\
4,3 \\
3,7\end{array}$ \\
\hline $\begin{array}{l}\text { Monatsmittel } \\
\text { Tägliche } \\
\text { Amplitude }\end{array}$ & $\begin{array}{l}\text { Mai } \\
\text { Juni } \\
\text { Juli } \\
\text { August }\end{array}$ & $\begin{array}{l}4,4 \\
1,6 \\
2,0 \\
2,0\end{array}$ & $\begin{array}{l}3,2 \\
1,4 \\
1,4 \\
2,7\end{array}$ & $\begin{array}{l}4,3 \\
1,8 \\
2,4 \\
2,4\end{array}$ & $\begin{array}{l}3,8 \\
0,9 \\
1,6 \\
2,6\end{array}$ & & $\begin{array}{l}2,7 \\
1,7 \\
1,0 \\
1,8\end{array}$ & $\begin{array}{l}3,4 \\
3,8 \\
1,8 \\
2,2\end{array}$ & $\begin{array}{l}3,6 \\
1,6 \\
0,8 \\
1,2\end{array}$ & $\begin{array}{l}3,4 \\
2,0 \\
1,1 \\
1,8\end{array}$ & $\begin{array}{l}3,8 \\
6,0 \\
2,3 \\
3,6\end{array}$ & $\begin{array}{l}2,8 \\
2,5 \\
1,8 \\
2.0\end{array}$ \\
\hline $\begin{array}{l}\text { Monatsmittel } \\
\text { Aperiodische } \\
\text { Tagesvariation }\end{array}$ & $\begin{array}{l}\text { Mai } \\
\text { Juni } \\
\text { Juli } \\
\text { August }\end{array}$ & $\begin{array}{l}4,6 \\
3,1 \\
3,1 \\
3,0\end{array}$ & $\begin{array}{l}3,9 \\
2,1 \\
3,1 \\
2,2\end{array}$ & $\begin{array}{l}4,4 \\
2,9 \\
2,6 \\
3,1\end{array}$ & $\begin{array}{l}4,2 \\
4,1 \\
4,2 \\
3,2\end{array}$ & & $\begin{array}{l}3,0 \\
2,4 \\
3,0 \\
2,2\end{array}$ & $\begin{array}{l}4,3 \\
2,8 \\
3,2 \\
2,1\end{array}$ & $\begin{array}{l}2,6 \\
2,6 \\
3,1 \\
3,3\end{array}$ & $\begin{array}{l}3,0 \\
2,1 \\
2,1 \\
1,3\end{array}$ & $\begin{array}{l}5,5 \\
3,2 \\
4,5 \\
2,7\end{array}$ & $\begin{array}{l}2,7 \\
3,7 \\
2,5 \\
1,7\end{array}$ \\
\hline
\end{tabular}


Fig. 2 Monatsmittel der Lufttemperatur u. der täglichen Amplltude $\rightarrow$ Tundrastationen - Gletscherstationen

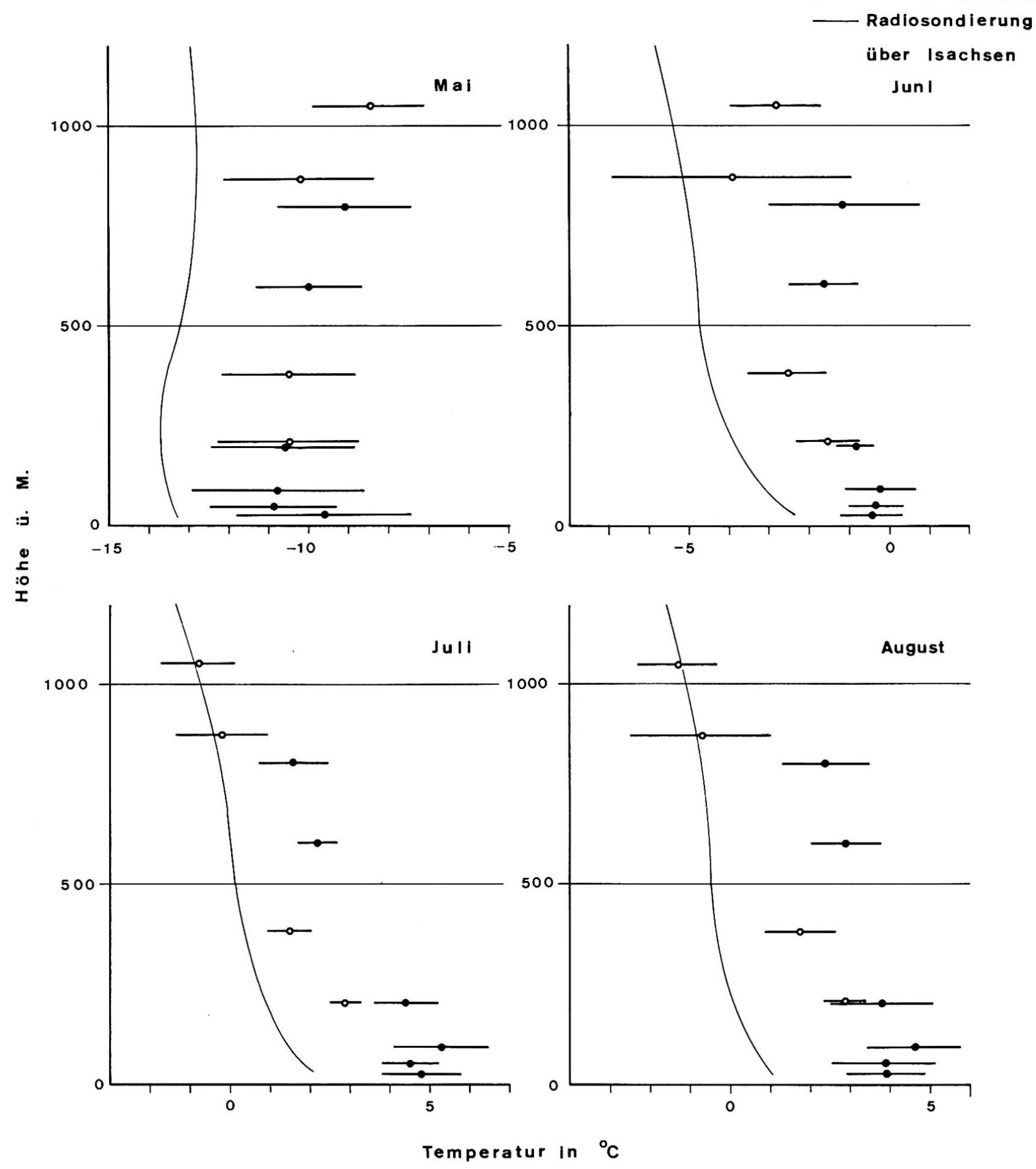


Es bieten sich zwei mögliche Wärmequellen an: latente Wärme aus der Kondensation in Wolken und die Erwärmung der Luft durch Wärmeabgabe aus der durch Strahlung erwärmten Schneedecke. Die erste Möglichkeit muß ausgeschieden werden wegen geringerem Niederschlag. Im Jahre 1969 betrug der mittlere fühlbare Wärmefluß im Monat Mai beim Base Camp $40 \mathrm{cal} \mathrm{cm}^{-2} \mathrm{Tag}^{-1}$ (OHMURA, 1972) und 1970 $20 \mathrm{cal} \mathrm{cm}^{-2} \mathrm{Tag}^{-1}$ (OHMURA und MÜLLLR, 1976). Der Durchschnitt der zwei Jahre ergibt für den Monat Mai einen fühlbaren Wärmefluß von ca. $30 \mathrm{cal} \mathrm{cm}^{-2} \mathrm{Tag}^{-1}$, was genügt, um die Temperatur der untersten $100 \mathrm{~m}$ der Atmosphäre um $13^{\circ} \mathrm{C}$ zu erwärmen. Infolge von Konvektion, Diffusion und Abkühlung der Atmosphäre durch langwellige Strahlung tritt diese Temperaturerhöhung nicht ein. Hingegen wirkt die fühlbare Wärme, die aus der Schneeoberfläche austritt, als die einzige grundlegende Wärmequelle für die Atmosphäre.

Trotz der erwähnten Homogenität der Temperaturen im Mai sind doch geringfügige Abweichungen zu beobachten. Die wärmste Gegend ist der oberste Teil des Baby Glacier auf $1050 \mathrm{~m}$ ü. M., während der Sander das kälteste Gebiet zu sein scheint. Der Temperaturunterschied zwischen diesen zwei Stationen beträgt $2,3^{\circ} \mathrm{C}$. Höhere Temperaturen wurden bei Stationen mit weit offener Umgebung beobachtet, so beim Fjord, auf der Ermine Ridge und beim Baby Glacier. Diese drei Stationen weisen im Mai eine längere potentielle Sonnenscheindauer auf als die übrigen. Diese befinden sich in Muldenlagen; ihre Temperaturen sind ca. $1,5^{\circ} \mathrm{C}$ niedriger als der ersten Gruppe. Zusätzlich zur lokalen Erwärmung im Mai durch Sonnenenergie ist oberflächliches Abfließen von gekühlter Luft für diese geringfügigen Temperaturabweichungen verantwortlich. Inversionen sind im Monat Mai weit häufiger als Fälle der Temperaturabnahme mit der Höhe. Der Grund dafür ist die Einwirkung von Stabilität auf den fühlbaren Wärmeeinfluß auf der Erdoberfläche. Im Mai treten intensive Inversionen selbst bei Hochdrucklagen und insbesondere zu Zeiten tiefen Sonnenstandes auf. Unter solchen Verhältnissen wirken das Tal des Wolf River und dasjenige des White Glacier als Abflußkanäle für kalte Luft. Die Verteilung der Luftemperaturen im Mai wird daher hauptsächlich durch das Relief bestimmt. Die Höhe über Meer und die Art der Oberfläche spielen in dieser Situation eine untergeordnete Rolle.
Die Monatsmittel der täglichen Amplitude der Lufttemperatur im Mai sind bei allen Stationen sehr ähnlich, was auf die übereinstimmende Strahlungswirkung zurückzuführen ist.

Im Juni werden die regionalen Abweichungen der Temperaturverhältnisse ausgeprägter. Erstens macht sich zu dieser Zeit eine Abhängigkeit von den Oberflächeneigenschaften bemerkbar. Die Temperaturdifferenz zwischen Tundra- und Gletschermeßstellen beträgt auf $200 \mathrm{~m}$ ü. M. $0,7^{\circ} \mathrm{C}$, wächst mit steigender Höhe und erreicht auf $800 \mathrm{~m}$ ü. M. ihre höchsten Werte während des ganzen Sommers, nämlich $3^{\circ} \mathrm{C}$. Über der Tundra nimmt die Temperatur in den untersten $500 \mathrm{~m}$ um $0,30^{\circ} \mathrm{C} / 100 \mathrm{~m}$ zu; über dem $500 \mathrm{~m}$ Niveau steigt sie hingegen um $0,10^{\circ} \mathrm{C} / 100 \mathrm{~m}$ an. Andrerseits nimmt die Temperatur auf den Gletschern bis auf $900 \mathrm{~m}$ um $0,38^{\circ} \mathrm{C} / 100 \mathrm{~m}$ aḅ. Drei Gründe können als Ursache für diese Temperaturabweichung angegeben werden. Erstens findet in den tieferliegenden Gebieten während eines beträchtlichen Teils des Monats Juni Schmelze statt. Während dieser Periode sind die Temperaturen über den Oberflächen von Tundra, Gletscher und Meereis sehr ähnlich; der regionale Durchschnitt über der Tundra ist am höchsten. Diese wenn auch nur geringe Differenz rührt von der Existenz von schneefreien Flecken her, deren Temperatur über dem Gefrierpunkt liegt. Zweitens wurde bei den wöchentlichen Besuchen auf Ermine Ridge im Juni 1969 und 1970 festgestellt, daß die Albedo entlang eines Schneekurses im Durchschnitt aus vier Messungen $48 \%$ betrug, während bei zwei Meßreihen bei der Moraine Station auf dem Gletscher auf etwa gleicher Höhe ein Wert von $71 \%$ gefunden wurde. Diese Albedodifferenz kann in den höheren Lagen als Hauptursache für die bedeutenden Temperaturunterschiede zwischen Tundra und Gletscher wirken. Der dritte und wahrscheinlich wichtigste Grund ist das häufige Vorkommen einer Stratusschicht zwischen $300 \mathrm{~m}$ und $600 \mathrm{~m}$ ü. M. die Sonnenscheindauer beim Base Camp ist im Juni geringer als im Mai, Juli und August. Daraus resultiert der geringe Temperaturkontrast zwischen Tundra und Gletscher in den tiefer liegenden Gebieten, während die über $600 \mathrm{~m}$ liegenden Gebiete von diesem Effekt frei sind.

Im Juni erreicht die tägliche Amplitude der Lufttemperatur der meisten permanent besetzten Stationen in der Arktis ein Minimum. Diese Feststellung gilt nur für Stationen unterhalb $300 \mathrm{~m}$ ü. M. und ist durch die 
Schmelze auf den häufigen Stratus zu erklären. Über $300 \mathrm{~m}$ ü. M. ist das Gegenteil der Fall. Es ist bemerkenswert, daß tagsüber die höchsten Temperaturen auf der Ermine Ridge, die tiefsten aber bei etwa $500 \mathrm{~m}$ ü. M. gemessen werden. Während der Nacht verursacht die starke Strahlungsabkühlung an der Oberfläche in hohen Lagen eine Temperaturabnahme. Um Mitternacht beträgt der Temperaturgradient über der Tundra $0,25^{\circ} \mathrm{C} / 100 \mathrm{~m}$ und über dem White Glacier $0,67^{\circ} \mathrm{C} /$ $100 \mathrm{~m}$.

Die Stationen bei Fjord und auf dem Sander des Gletschervorfeldes zeigen im Vergleich zu den anderen Tundrenmeßstellen leicht tiefere Temperaturen, was durch die Wirkung der nahegelegenen, kühleren Oberflächen des Expedition Fjord, resp. des White Glacier zu erklären ist. Diese Tendenz ist in den darauffolgenden Monaten noch stärker ausgeprägt.

Wie im Mai ist die Juni-Lufttemperatur entlang der Berghänge verglichen mit jener der freien Atmosphäre auf gleichem Niveau wesentlich höher. Die Tundrengebiete, insbesondere oberhalb $500 \mathrm{~m}$, zeigen die größten Abweichungen von den Temperaturen der freien Atmosphäre. Die fast einzige Wärmequelle ist der fühlbare Wärmefluß von der Oberfläche, der im Juni beim Base Camp auf zirka $90 \mathrm{cal} \mathrm{cm}^{-2} \mathrm{Tag}^{-1}$ ansteigt (OHMURA, 1972, OHMURA und MÜLLER, 1976).

Der Juli ist auf den Tundra-Stationen, die nahe am Meeresspiegel liegen, der wärmste Monat des Jahres. Der Juli-Temperatur-Gradient über der Tundra erreicht $0,80^{\circ} \mathrm{C} / 100 \mathrm{~m}$ bis zu Höhen von $500 \mathrm{~m}$ ü. M. Jahreshöchstwerte. Unterhalb $500 \mathrm{~m}$ beträgt er auf Gletschern $0,75^{\circ} \mathrm{C} / 100 \mathrm{~m}$. Oberhalb $500 \mathrm{~m}$ bleibt er hingegen sowohl über Tundra als auch über Gletschern mit $0,33^{\circ} \mathrm{C} / 100 \mathrm{~m}$ gering. Die Temperaturdifferenz zwischen Tundra- und Gletscherstationen ähnlicher Höhenlage ist bei $1,5^{\circ} \mathrm{C}$ von der Gletscherzunge bis auf $800 \mathrm{~m}$ beinahe konstant. Charakteristisch für den Monat Juli ist ferner die Zunahme der täglichen Amplitude über der tiefer liegenden und eine markante Abnahme derselben über der höher liegenden Tundra. Dieses Phänomen hat seine Ursache hauptsächlich in der Austrocknung der Tundraoberfläche der geringen Höhen, sowie in der immer noch andauernden Schneeschmelze der feuchten Tundraoberfläche in höheren Lagen. Die kleinste tägliche Amplitude über der Tundra wurde im Juli im Talboden des oberen Wolf River Valley gemessen.

Die tägliche Amplitude über den Gletschern nimmt im allgemeinen mit steigender Höhe stetig zu, was auf die geringe Schmelze in den höheren Lagen zurückzuführen ist. Beim Fjord und auf dem Sander wurden im Vergleich zu den anderen Tundrastationen tiefere Temperaturen registriert. Die Monatsmitteltemperaturen dieser zwei Meßstellen sind denjenigen von Gletscherstationen ähnlich, hingegen unterscheiden sie sich von diesen durch größere tägliche Amplituden.

Im August tendiert die vertikale Verteilung der Lufttemperatur über der Tundra wiederum gegen isotherme Verhältnisse, während diejenige über den Gletschern von der Zunge bis zur Obergrenze des Untersuchungsgebietes immer noch stark abnehmend bleibt. Dadurch wird die Temperaturdifferenz zwischen Tundra- und Gletscherstationen wie im Juni mit zunehmender Höhe größer. Der Grund liegt hauptsächlich in der Erwärmung der hoch liegenden Tundraböden. In der über $500 \mathrm{~m}$ ü. M. gelegenen Tundra werden üblicherweise im August die höchsten Temperaturen des Jahres gemessen, weil dann die Erdoberfläche die größte Ausaperung erreicht.

Die tägliche Amplitude der Lufttemperatur über der Tundra wird im August verglichen mit dem Juli wieder größer, was durch die einsetzenden Tag- und Nachtunterschiede in der Sonnenstrahlung und durch die Austrocknung der Bodenoberfläche zu erklären ist. Die Größe der täglichen Amplitude ist in Bezug auf die Höhe konstant. Das wird mit der Homogenität der Tundraoberfläche erklärt, die dann normalerweise in allen Höhenbereichen schneefrei und trocken ist. Auf Gletschern bleibt die Amplitude in tiefen Lagen klein, wächst aber mit steigender Höhe, was darauf hindeutet, daß die Schmelze in höheren Lagen rasch abnimmt.

Die wichtigsten Elemente der Verteilung der Monatsmittel von täglicher Maximal- und Minimaltemperatur können wie folgt zusammengefaßt werden:

Im Mai und Juni zeigt die Tundrameßstelle auf $800 \mathrm{~m}$ ü. M. (Ermine Ridge) die höchste tägliche Maximaltemperatur der Gegend. Im Juli wird das höchste mittlere tägliche Maximum in der Nähe des Fjords anzutreffen sein, während es im August über tief liegender Tundra, aber in möglichst großer Entfernung von Fjord und Gletschern liegt. Die tiefsten Monatsmittel der täglichen Maximaltemperatur werden im Mai und Juni in Talsohlen registriert, während sie im Juli/August in größeren Höhen und in Gletschernähe erscheinen. 
Hinsichtlich der Monatsmittel der täglichen Minimaltemperaturen ist im Mai der Talboden des Wolf River Valley am wärmsten, während die kälteren Stellen sich auf tief liegender Tundra befinden. Im Juni ist der Sander das wärmste Gebiet und die tiefsten Temperaturen werden oberhalb $400 \mathrm{~m}$ gefunden. Im Juli fällt die tägliche Minimaltemperatur vom Meeresspiegel bis auf $800 \mathrm{~m}$ stetig ab. Dieselbe Tendenz hält auch im August an.

Das Monatsmittel der aperiodischen Tagesvariation ist als Differenz zwischen dem Monatsmittel der Tagesschwankung und dem Monatsmittel der täglichen Amplitude definiert. Letztere ist ein Maß für die Wirkung der Strahlung an der Oberfläche, während die mittlere aperiodische Tagesvariation ein geeignetes $\mathrm{Maß}$ für die advektive Temperatur darstellt. Tab. 1 zeigt, daß diese Größe für alle Stationen ziemlich konstant ist. Werden die vier Sommermonate gemittelt, sind die Werte beim Sander und dem Wolf River kleiner als bei den anderen Stationen. Diese zwei Meßstellen liegen in Vertiefungen, die die Advektion stören. Im Monatsmittel ist die aperiodische Tagesvariation im Mai am größten. Das ist auf die Tatsache zurückzuführen, daß der Mai der einzige Monat ist, der eine isotherme Schichtung in der freien Atmosphäre aufweist. Das bedeutet, daß der vertikale Gradient der potentiellen Temperatur von den vier untersuchten Monaten im Mai am größten ist. In gebirgigen Gegenden, wie in diesem Untersuchungsgebiet, geschieht der Austausch von Luft, der zu großen Temperaturänderungen führt, am häufigsten zwischen der Oberfläche und höheren Schicht, anstatt durch horizontale Advektion. Daher zeigen Monate mit einem starken vertikalen Gradienten der potentiellen Temperatur eine größere aperiodische Tagesschwankung. In diesem Sinn sollte das größte Monatsmittel der aperiodischen Tagesvariation mitten im Winter gefunden werden, wenn die Inversionen am stärksten sind. Dieser Gedankengang wird durch Loewe's Daten von der grönländischen Station unterstützt (LOEWE, 1935).

\section{Frostfreie Tage und frostfreie Periode}

Auch in der Arktis ist das Wachstum und die Reproduktion von Pflanzen frostempfindlich. Die regionale Verteilung der frostfreien Tage wird aufgrund der Temperaturen im Wetterhüttenniveau bestimmt, die

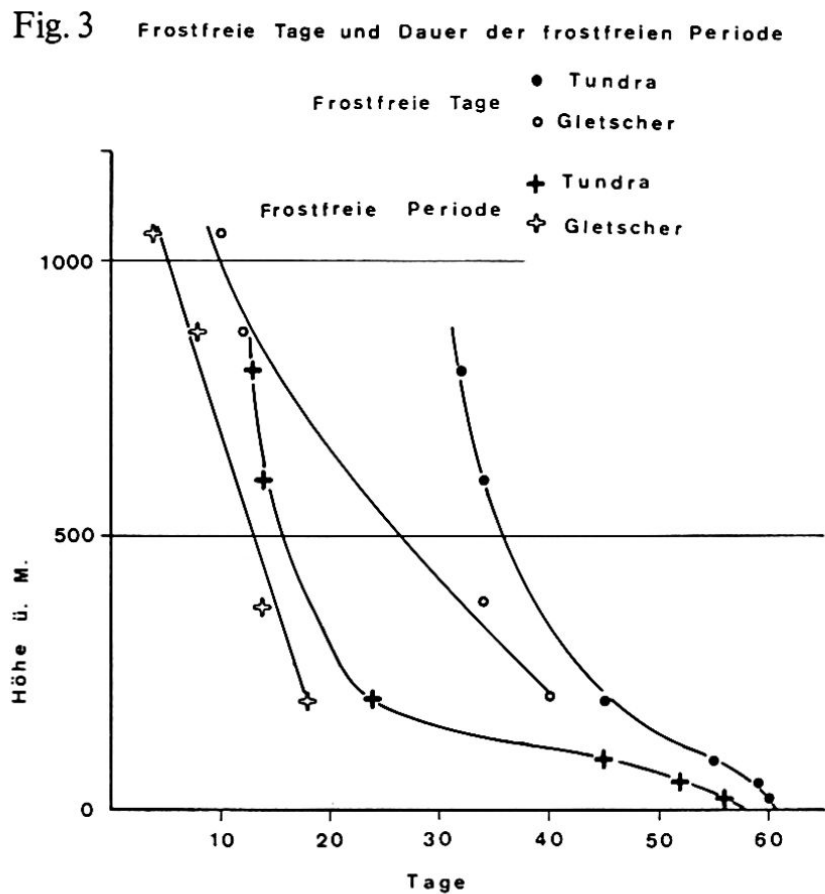

1969, 1970 und 1972 gemessen wurden. Das Ergebnis ist in Fig. 3 dargestellt. Die größte Anzahl von frostfreien Tagen wird in der Nähe des Fjordes gefunden, obwohl die Monatsmitteltemperaturen dort tiefer liegen als weiter im Landesinnern. Der Grund dafür ist, $\mathrm{da} ß$ die täglichen Minimaltemperaturen an dieser Stelle im Juli und August am höchsten liegen. Die Anzahl der frostfreien Tage nimmt zwischen 100 und $200 \mathrm{~m}$ ü. M. stark ab und fällt darüber mit steigender Höhe viel allmählicher. Sie beträgt bei Fjord 60 Tage und verringert sich bis auf $800 \mathrm{~m}$ auf die Hälfte. Es ist bemerkenswert, daß der Unterschied in der Anzahl der frostfreien Tage zwischen Tundra und Gletscher unterhalb $400 \mathrm{~m}$ sehr klein ist, hingegen nimmt die Differenz oberhalb $500 \mathrm{~m} \mathrm{zu}$. Dadurch wird angedeutet, daß Pflanzen keine nennenswerte Kürzung der frostfreien Tage erleiden, wenn sie unterhalb $400 \mathrm{~m}$ in der Nähe von Gletschern wachsen. Diese Feststellung wird durch die große Zahl frostfreier Tage im Sander, die derjenigen am Ende des Fjordes beinahe gleichkommt, bestätigt. Oberhalb $400 \mathrm{~m}$ hingegen müssen die Pflanzen eine starke Beschneidung der Anzahl der frostfreien Tage in Kauf nehmen, wenn sie sich in der Nähe der Gletscher ansiedeln.

Die Dauer der frostfreien Periode wird verkürzt, wenn Temperaturen unter dem Gefrierpunkt trotz einer 
großen Zahl frostfreier Tage häufig auftreten. Das ist auf der Tundra oberhalb $200 \mathrm{~m}$ der Fall. Wenn die tägliche Minimaltemperatur im Juni über der Tundra zwischen dem Meeresniveau und $100 \mathrm{~m}$ einmal über $0^{\circ} \mathrm{C}$ ansteigt, fällt sie bis in den August nur sehr selten auf den Gefrierpunkt zurück. Darum ist in der tiefst liegenden Tundra die Dauer der frostfreien Periode nicht sehr verschieden von der Anzahl der frostfreien Tage. Die Dauer der frostfreien Periode ist bis $200 \mathrm{~m}$ ü. M. stark höhenabhängig und dauert auf diesem Niveau nur noch 24 Tage, verglichen mit 56 Tagen beim Fjord. Oberhalb $300 \mathrm{~m}$ beträgt sie weniger als 20 Tage und bleibt fast höhenunabhängig. Die frostfreie Periode dauert auf Ermine Ridge nur 13 Tage, was weniger als ein Viertel vom Wert beim Fjord ist. Die Differenz in der Länge der frostfreien Periode zwischen Tundra und Gletschern beträgt auf $200 \mathrm{~m}$ 6 Tage und auf $800 \mathrm{~m} 4$ Tage. In allen Höhenbereichen hat die Nähe von Gletschern einen geringen Einfluß auf die Dauer der frostfreien Periode.

\section{Frostwechseltage}

Der Gefrier-Tau-Zyklus ist von Wichtigkeit sowohl für die Geomorphologie als auch die Pedologie und die Biologie. Als $\mathrm{Ma} B$ für den Gefrier-Tau-Zyklus wird die Anzahl der Frostwechseltage angegeben. Das Jahrestotal der Frostwechseltage ist in Fig. 4 dargestellt. Es ist unterhalb $100 \mathrm{~m}$ ü. M. mit $20 \mathrm{Tagen} / \mathrm{Jahr}$ sehr klein und nimmt gegen $200 \mathrm{~m}$ ü. M. stark zu. Oberhalb $200 \mathrm{~m}$ wächst es weniger rasch und erreicht auf

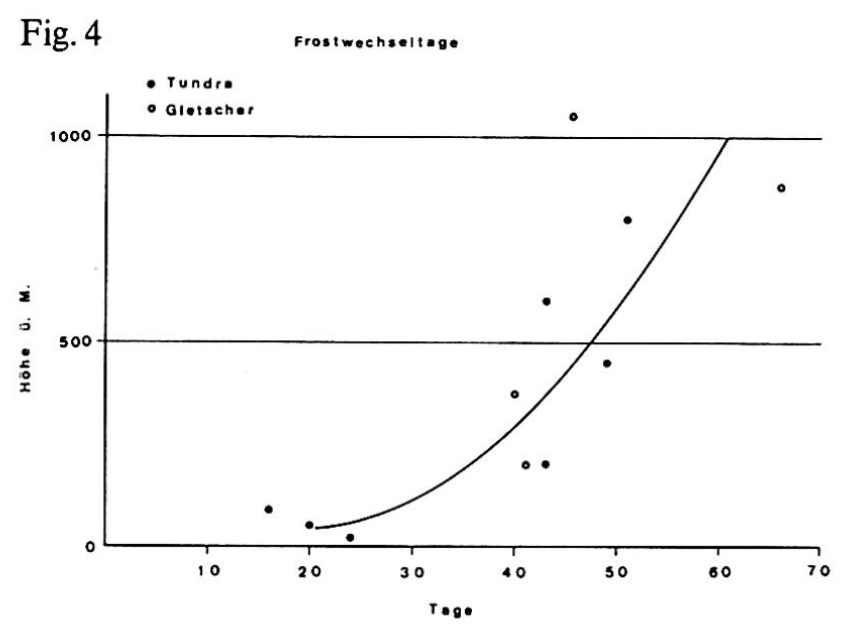

der Tundrameßstelle auf 800 m 51 Tage/Jahr. Die Differenz in der Anzahl der Frostwechseltage zwischen Tundra- und Gletscherstationen scheint unbedeutend zu sein.

Im Zusammenhang mit den Prozessen auf und unter der Bodenoberfläche ist es von Bedeutung, daß der Gefrier-Tau-Zyklus im allgemeinen nur während der schneefreien Periode wirksam ist. Auf der Tundra unterhalb $100 \mathrm{~m}$ treten die meisten Frostwechseltage während der Schmelzsaison auf, und nur 5 bis 7 Tage/ Jahr entfallen auf die schneefreie Periode. Oberhalb $200 \mathrm{~m}$ hingegen gibt es im Juni, Juli und August fast gleichmäßig je ca. 15 Frostwechseltage pro Monat. Das bedingt zwischen $200 \mathrm{~m}$ und $600 \mathrm{~m}$ ü. M. eine Zone mit maximaler Anzahl von Frostwechseltagen während der schneefreien Periode, nämlich mit 29 Tagen/Jahr auf $200 \mathrm{~m}$ und 30 Tagen/Jahr auf $600 \mathrm{~m}$. Oberhalb dieser Zone reduziert eine lang andauernde Schneebedeckung die Anzahl der Frostwechseltage während der schneefreien Periode. Auf $800 \mathrm{~m}$ sind es nur noch 18 Tage. Die Anzahl der Frostwechseltage ist in diesem Gebiet beträchtlich kleiner als in südlicheren Regionen von Kanada. Der Befund von FRASER (1959), daß Gefrier-Tau-Zyklus in Kanada von Süden nach Norden seltener werden, wird durch die Beobachtungen auf Axel Heiberg bestätigt. Hingegen muß die weitverbreitete Auffassung, die besonders auch von JENNESS (1952) vertreten wird, daß Gefrier-Tau-Zyklen in der Arktis zwischen Mai und Oktober fast täglich stattfinden und ein eminent wichtiges Phänomen für die Prozesse auf der Tundra seien, bezweifelt werden.

\section{Niederschlag}

Das dreijährige Mittel der monatlichen Niederschlagsmengen ist in Fig. 5 gegen die Höhe aufgetragen. Im allgemeinen nimmt die Niederschlagsmenge im untersuchten Gebiet mit der Höhe linear zu, ausgenommen auf Ermine Ridge. Diese Station liegt im obersten Teil eines breiten Rückens und dürfte dem LettauEffekt unterworfen sein. Die Rate der Niederschlagszunahme mit der Höhe ist proportional zur Niederschlagsmenge. Die Regressionsgerade wurde für die normalisierte Niederschlagsmenge P/Po und die Höhe $\mathrm{H}$ berechnet, wobei $\mathrm{P}$ den Niederschlag auf der Höhe $\mathrm{H}$ und $\mathrm{Po}$ dieselbe Größe auf Meeresniveau bezeichnet. $\mathrm{H}$ ist die Höhe über dem Meeresspiegel und wird in 
Fig. 5 monots- und sommertiotel dere Niederschioge in $\mathrm{mm}$

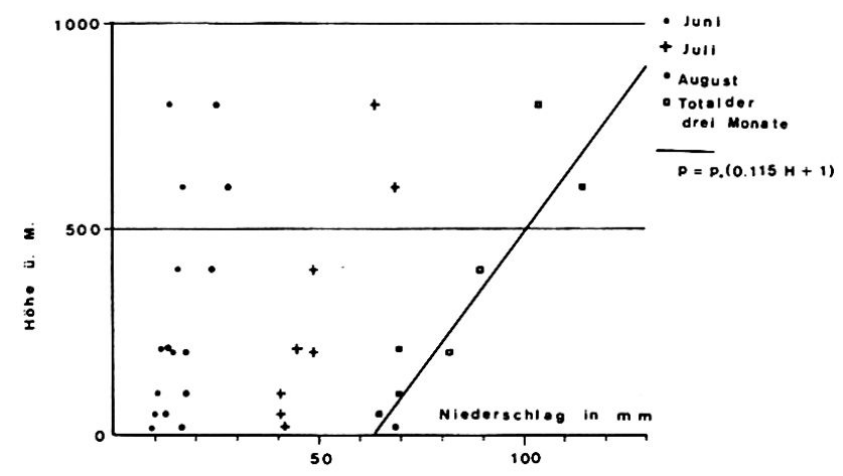

der Einheit von $100 \mathrm{~m}$ angegeben. Der Grund für die Wahl des Niederschlags auf Meeresniveau als Basis für die Normalisierung ist die Tatsache, daß die meisten langfristigen Niederschlagsmengen in der Arktis nahe am Meeresniveau gemacht werden. Die gewonnene Beziehung $\mathrm{P}=\mathrm{Po}(0,115 \mathrm{H}+1)$ ist unabhängig von der Beobachtungsdauer, hat aber über lange Perioden hinweg größere Bedeutung. Auf Stationen, die vom Lettau-Effekt beeinflußt sind, sollte man sie nicht anwenden. Daher wurde auch Ermine Ridge nicht in die Berechnungen einbezogen. Eine weitere Einschränkung dieser Beziehung wird durch die Orographie des Gebietes gegeben. Das gegenwärtige Resultat basiert auf Daten, die auf der Westseite der Insel gesammelt wurden. Seine Gültigkeit in anderen Gebieten wurde noch nicht untersucht. Es ist besonders wichtig zu erwähnen, daß die Niederschlagsmengen auf dem zentralen Teil der McGill Eiskappe deutlich von denjenigen in den Küstengebieten abweichen (ANDREWS, 1964, HAVENS, 1964, MÜLLER and ROSKINSHARLIN, 1967).

Die vorliegende Arbeit wurde finanziell durch den National Research Council of Canada (durch Grant No. A-2662 an Dr. F. Müller), die McGill Universität Montreal sowie das Geographische Institut der ETH Zürich unterstützt. Atmospheric Environment Canada stellte Instrumente zur Verfügung; die wertvolle Unterstützung Gordon A. McKay, Direktor der Meteorological Applications Branch wird bestens verdankt. Das Polar Continental Shelf Project der kanadischen Regierung gewährte die so wichtige logistische Hilfe.

\section{Literatur}

ANDREWS, R. H. 1964. Meteorology and Heat Balance of the Ablation Area, White Glacier, Canadian Arctic Archipelago - Summer 1960. Axel Heiberg Island Research Reports, Meteorology, No. 1, McGill University, Montreal.

FRASER, J. K. 1959: Freeze-thaw frequencies and mechanical weathering in Canada. Arctic, Vol. 12, No. 1, p. $40-53$.

HAVENS, J. M. 1964. Meteorology and Heat Balance of the Accumulation Area, McGill Ice Cap, Canadian Arctic Archipelago - Summer 1960. Axel Heiberg Island Research Reports, Meteorology, No. 2, McGill University, Montreal.

JACKSON, C. I. 1959. Coastal and inland weather contrasts in the Canadian arctic. Journ. Geophys. Res., Vol. 64, No. 10, p. 1451-1455.

JENNESS, J. L. 1952. Erosive forces in the physiography of the western arctic islands. Geogr. Rev., Vol.42, p. 238-252.

LOEWE, F. 1935. Das Klima des grönländischen Inlandeises. Köppen-Geiger, Handbuch der Klimatologie K., Verlag Gebrüder Borntraeger, Berlin.

MÜLLER, F. and N. ROSKIN-SHARLIN, 1967: A High Arctic Climate Study on Axel Heiberg Island, Canadian Arctic Archipelago - Summer 1961. Axel Heiberg Island Research Reports, Meteorology, No.3, McGill University, Montreal.

MÜlleR, F. et al. 1961. Axel Heiberg Island, Preliminary Report 1959-1960. McGill University, Montreal.

MỨlleR, F. et al. 1963. Axel Heiberg Island, Preliminary Report 1961-1962. Axel Heiberg Island Research Reports, McGill University, Montreal.

OHMURA, A., 1972. Heat and water balance on arctic tundra. International Geography 1972, p. 175-176.

OHMURA, A. and F. MÜLLER. 1976. Heat balance measurement on arctic tundra, Axel Heiberg Island, Canadian Arctic Archipelago. International Geography 1976, Vol. 2, p. 80-84. 
Abb.1 Temporäre Feldstation im Akkumulationsgebiet, White Glacier, Axel Heiberg Island, N.W.T.; Massenhaushalts- und Firntemperaturmessungen sowie Klimastudien. Foto: F. Müller

Abb. 2 Automatische Wetterstation (Ott und Rauchfuss Modell kombiniert) nahe der Gleichgewichtslinie auf dem White Glacier. Foto: K. Steffen
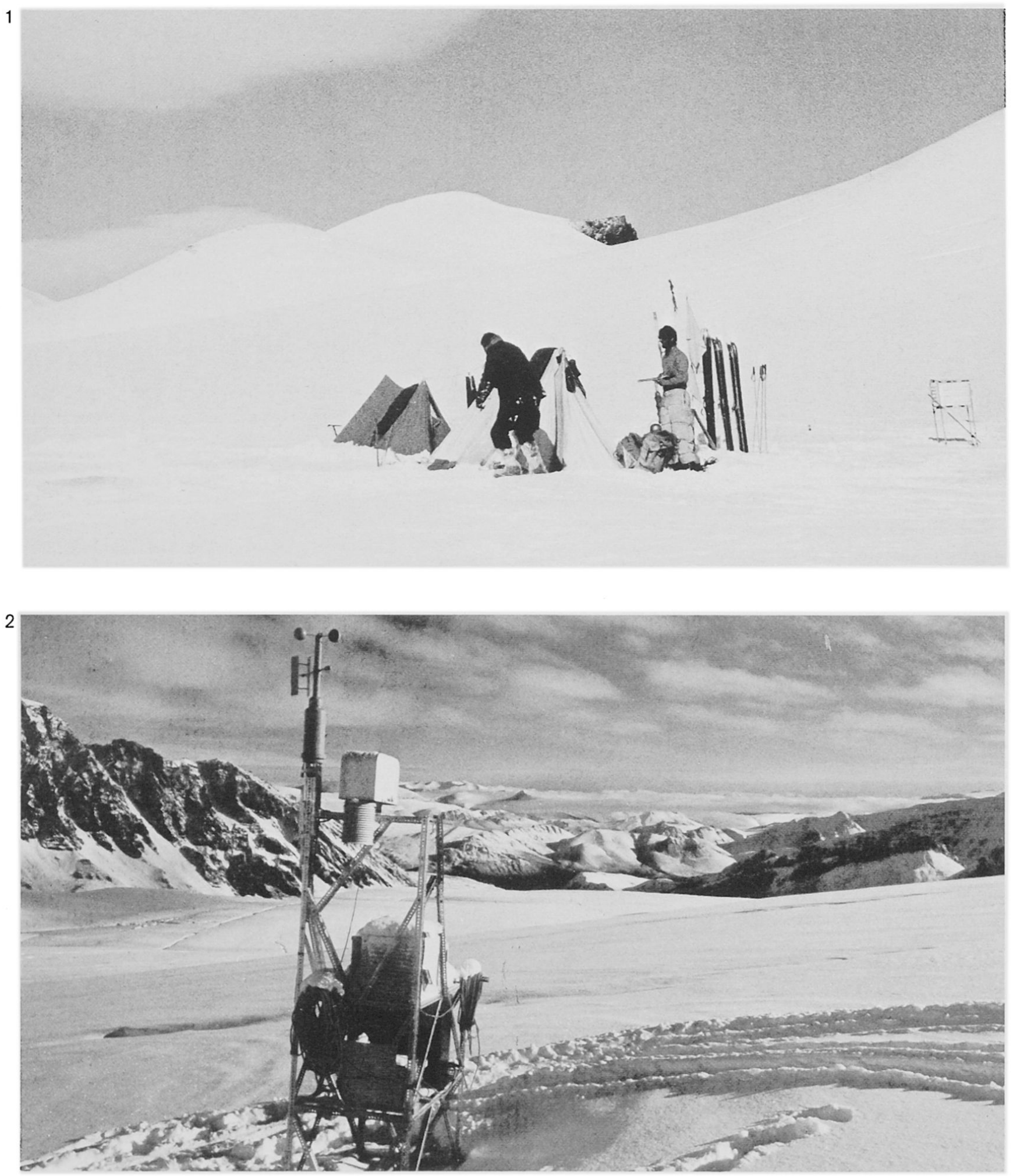